\title{
Isolated Tubercular Osteomyelitis of Scapula - A Report of Two Cases and Review of Literature
}

\author{
Gopisankar Balaji ${ }^{1}$, Justin Arockiaraj ${ }^{2}$, Alfred Cyril Roy ${ }^{2}$, Anand Ashok ${ }^{2}$
}

\author{
What to Learn from this Article? \\ Presentation and Diagnosis of tuberculous lesions of Scapula.
}

Abstract

Introduction: Isolated tuberculosis of the scapula is rare. The presentation mimics the tumors of scapula. Hence it is important to know the clinical presentation and when to suspect tubercular osteomyelitis of scapula. Few cases have been described before. We have analyzed all reports with isolated scapular tuberculosis so far and described the varied presentations.

Case Report: We report two cases of isolated scapular tuberculosis. One patient underwent aspiration and was started on antituberculous drugs ( ATT) and the other underwent debridement, drainage of abscess and then was started on ATT. Both had excellent outcomes at the end of one year follow up. We reviewed previous published literature of isolated scapular tuberculosis. The most common presentation of scapular tuberculosis is as follows: age less than 30 years and there is no difference with respect to gender. Swelling and pain are the commonest symptoms. Lytic areas with surrounding sclerosis is the commonest radiological finding. Body of the scapula is affected most commonly. Prognosis is excellent with adequate treatment.

Conclusion: To conclude, clinicians should have high index of suspicion for diagnosing this condition as it mimics tumors. Pain and swelling is the most common presentation of scapular tuberculosis. Radiography shows lytic lesion with marginal sclerosis. The prognosis is excellent with appropriate treatment.

Keywords: Tuberculosis, osteomyelitis, scapula.

Author's Photo Gallery
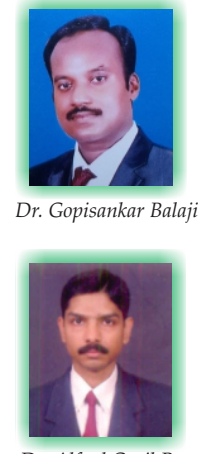

Dr. Alfred Cyril Roy
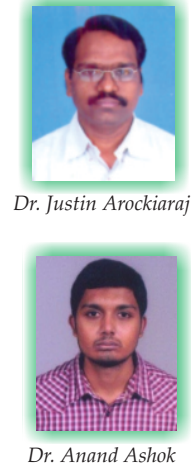

${ }^{1}$ Department of Orthopaedics, Jawaharlal Institute of Postgraduate Medical Education and Research, Pondicherry. India.

2Department of Orthopaedics Unit 1, Christian Medical college, Vellore. India.

Address of Correspondence

Dr. Gopisankar Balaji .G.

Assistant Professor,Department of Orthopaedics, Jawaharlal Institute of Postgraduate Medical Education and Research

Dhanvantari Nagar, Pondicherry- 605006. India.

Email: drgopi9596@gmail.com

\section{Introduction}

Tuberculosis is the major cause of skeletal infection in the developing countries. Skeletal tuberculosis accounts for 10 - $35 \%$ of extra pulmonary tuberculosis and only $2 \%$ of all cases of tuberculosis [1]. Less than one percent of skeletal tuberculosis occurs in the shoulder [2]. Occurrence of tuberculosis in flat bones without any other foci is very rare. We treated two patients with tuberculosis of scapula successfully. We reviewed all the previous reports of scapular tuberculosis in English literature to analyse the common clinical presentation, the site of occurrence in scapula and radiographic appearance.

\section{Patient 1:}

Case Report

A 17-year-old male presented with dull aching pain and gradually progressive swelling over the left scapular region since five months. Pain was aggravated on shoulder movement and on lying supine. He had no fever. Physical examination revealed a swelling of 3 X $3 \mathrm{~cm}$ which was located in the left supraspinous region. The swelling was 


\begin{tabular}{|c|l|c|l|l|l|l|}
\hline S.No & \multicolumn{1}{|c|}{ Study } & Age/Sex & \multicolumn{1}{|c|}{ Location } & Side & Presenting Complaints & \multicolumn{1}{|c|}{ Treatment } \\
\hline 1 & Mohan et al 1991 [2] & $23 / \mathrm{F}$ & Body of scapula & Rt & Pain and swelling & Drainage \& ATT \\
\hline 2 & Gusati et al $1997[5]$ & NA & Spine of scapula & NA & Pain & Surgery \& ATT \\
\hline 3 & Vohra et al $1997[6]$ & NA & Body of scapula & NA & NA & NA \\
\hline 4 & Kam et al 2000 [7] & 33/M & Acromian & Rt & Pain Discharging sinus & $\begin{array}{l}\text { Debridement } \\
\text { curettage, Antibiotic } \\
\text { beads \& ATT }\end{array}$ \\
\hline 5 & Husen et al 2006 [8] & $18 / \mathrm{M}$ & $\begin{array}{l}\text { Spine of scapula near } \\
\text { neck }\end{array}$ & Lt & Pain & ATT \\
\hline 6 & Srivastav et al 2006 [9] & 26/F & Inferior angle & Lt & Pain and swelling & Aspiration \&ATT \\
\hline 7 & Solav 2007 [10] & $54 / F$ & $\begin{array}{l}\text { Spine of scapula and } \\
\text { medial margin }\end{array}$ & Lt & Pain & ATT \\
\hline 8 & Jain et al 2009 [3] & 14/M & $\begin{array}{l}\text { Body of scapula near } \\
\text { glenoid }\end{array}$ & Rt & $\begin{array}{l}\text { Pain, Swelling, discharging } \\
\text { sinus }\end{array}$ & ATT \\
\hline 9 & Singh et al 2009 [1] & 49/F & $\begin{array}{l}\text { Scapular body inferior } \\
\text { to spine }\end{array}$ & Lt & Pain and swelling & I\& D \\
\hline 10 & Tripathy et al 2010 [4] & 22/M & Scapular body & Rt & Pain and swelling & ATT \\
\hline
\end{tabular}

\begin{tabular}{|c|c|c|c|}
\hline S.No & \multicolumn{2}{|c|}{ ANALYSIS OF DATA } & PERCENTAGE \\
\hline \multirow{2}{*}{1} & \multirow{2}{*}{ AGE } & $<30$ years & $70 \%$ \\
\hline & & $>30$ years & $30 \%$ \\
\hline \multirow{2}{*}{2} & \multirow{2}{*}{ SEX } & Male & $50 \%$ \\
\hline & & Female & $50 \%$ \\
\hline \multirow{2}{*}{3} & \multirow{2}{*}{ Duration before presentation } & $<6$ months & $90 \%$ \\
\hline & & $>6$ months & $10 \%$ \\
\hline 4 & \multicolumn{2}{|c|}{ Presence of systemic signs } & $30 \%$ \\
\hline \multirow{4}{*}{5} & \multirow{4}{*}{ Anatomic location } & Body of scapula & $50 \%$ \\
\hline & & Spine of scapula & $25 \%$ \\
\hline & & Inferior angle & $17 \%$ \\
\hline & & Acromion & $8 \%$ \\
\hline \multirow{2}{*}{6} & \multirow{2}{*}{ Radiographic findings } & Osteolytic lesion & $100 \%$ \\
\hline & & Marginal sclerosis & $60 \%$ \\
\hline 7 & Treatment & Surgery-Debridement & $27 \%$ \\
\hline 8 & \multicolumn{2}{|c|}{ Results were good } & $100 \%$ \\
\hline 9 & \multicolumn{2}{|c|}{ Complications } & $0 \%$ \\
\hline 10 & \multicolumn{2}{|c|}{ Comorbidities } & $0 \%$ \\
\hline
\end{tabular}

Table 1 Review of literature of previously reported cases of Isolated Table 2: Analysis of all twelve patients with isolated scapula scapular tuberculosis

tuberculosis

warm and tender with soft cystic consistency. There was no discharging sinus. Terminal range of motion was restricted with pain especially during the last $15^{\circ}$ of abduction.

His blood investigations were unremarkable with ESR of $15 \mathrm{~mm} / \mathrm{hr}$. Anteroposterior radiograph of left shoulder revealed a well defined, $4 \times 3 \mathrm{~cm}$ osteolytic lesion at the superomedial aspect of body of scapula, with surrounding sclerosis [Fig.1]. The lateral radiograph showed increased soft tissue shadow in the superior aspect of scapula. His chest radiograph was normal. Since appearance was similar to a bone tumor, differentiation was important and work up on same lines was planned. Whole body bone scan showed abnormal increase in tracer uptake in the body of scapula with the rest of the skeleton being normal. Computer tomography ( CT ) showed erosions involving the supero-medial aspect of the body of scapula and

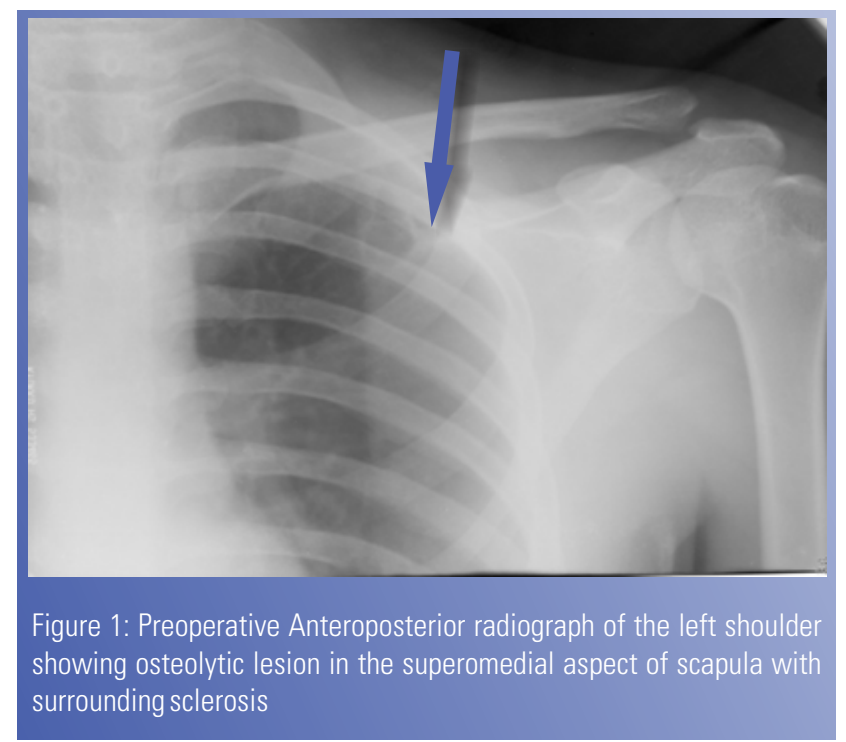

along the base of the spinous process of scapula [Fig. 2 and Fig.3 ]. Magnetic resonance imaging ( MRI ) scan revealed a well- defined lobulated lesion [ Fig.4 ]. It showed high signal on T2W and low signal on T1W images. Central T2W hypointensity was also seen. There was a soft tissue component extending posteriorly into the subcutaneous tissue. The left shoulder joint was normal. The differential diagnosis were chronic infection probably tuberculosis, Eosiniphilic granuloma or tumourous conditions. A CT guided biopsy was done Tissue and fluid were sent for cultures and histopathology. Biopsy revealed granulomas composed of epithelioid cells, lymphocytes and a few multinucleate giant cells suggestive of tuberculosis. Culture from Lowenstein Jensen medium showed acid fast bacilli after six weeks.

He was started on antitubercular therapy for a period of nine months. He was on two months of intensive therapy and seven months of continuation therapy. The dosage of the drugs given were Cap. Rifampicin $600 \mathrm{mg}$, Tab. Isoniazid $450 \mathrm{mg}$, Tab. Ethambutol $800 \mathrm{mg}$, Tab. Pyrazinamide $1250 \mathrm{mg}$, Tab. Pyridoxine $10 \mathrm{mg}$ on daily basis. He had complete resolution of symptoms within four months. At one year follow up, he had painfree full range of movements of shoulder .

\section{Patient 2:}

A 17-year old girl presented with swelling over her right upper back since three months. She had occasional pain. She had no fever. She had loss of appetite and weight loss. Physical examination revealed a $6 \mathrm{X} 7 \mathrm{~cm}$ soft,cystic and fluctuant swelling over the right scapular region [ Fig.5 ]. 


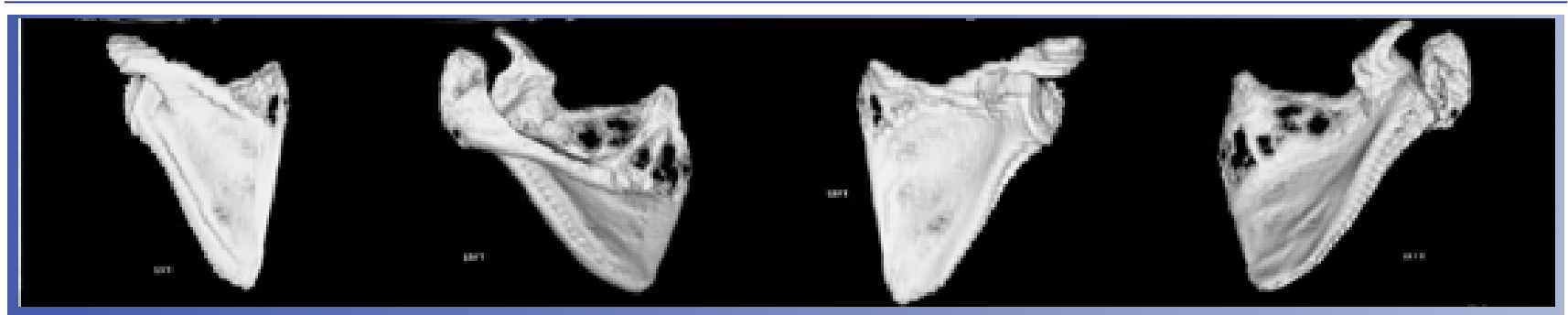

Figure 2: Computer tomograph 3D reconstruction images showing erosive lesions on the body of scapula around the spinous process.

There were no discharging sinuses. Cervical spine and shoulder range of motion were full and painless. Systemic examination was unremarkable. Blood investigations revealed hemoglobin of $10.3 \mathrm{gm} \%$, total count of 11,300 and the erythrocyte sedimentation rate was $97 \mathrm{~mm} / \mathrm{hr}$. Radiograph showed an osteolytic lesion in the inferior angle of scapula with surrounding sclerosis [Fig. 6]. Computer tomography of thorax showed a large well defined homogenously hypoechoic mass measuring about $63 \times 69 \mathrm{~mm}$ in the posterior chest wall ,abutting and infiltrating right trapezius, supraspinatus , infraspinatus and lattissimus dorsi, with extension into the right axilla. There were erosions and destruction of inferior angle of the right scapula with suspected sequetra [Fig. 7]. There was no intra thoracic extension. There were no parenchymal changes in right hemithorax or rib erosions. All these features were suggestive of abscess in the posterior chest wall with osteomyelitis of right scapula.Ultrasonography of the abdomen was normal.

She underwent incision and drainage of the abscess along with debridement, sequestrectomy and curettage of right scapula [Fig.8]. Tissue cultures and histopathology were done. Mycobacterial culture ( Lowenstein Jensen medium) demonstrated Acid Fast bacilli typical of Mycobacterium tuberculosis after six weeks which was sensitive to all the first line antituberculous drugs. Biopsy showed inflammatory granulation tissue and fibroadipose tissue containing discrete confluent granulomas composed of epithelioid histiocytes and
Langhans' type multinucleate giant cells surrounded by a dense infiltrate of lymphocytes, plasma cells, and aggregates of neutrophils. Multiple foci of caseous necrosis were present. She had identical treatment regimen as described earlier. She had complete resolution of symptoms and no recurrence at the end of three year follow up.

\section{Discussion}

Tuberculosis is a major health problem in the developing countries. Despite recent advances in its diagnosis and treatment, it has become a global epidemic with the emergence of HIV infection and multidrug resistance. Skeletal tuberculosis occurs secondary to hematogenous spread from primary focus which are usually pulmonary or lymph nodes.. Less than $1 \%$ of all skeletal tuberculosis affects the shoulder, a fraction of it involves the scapula [3]. Tuberculosis of the flat membranous bone is very rare. Very few cases of isolated scapular involvement have been reported in literature.

One of the difficulties in diagnosing skeletal tuberculosis is the non specific clinical presentation. Clinically, skeletal tuberculosis presents with pain and swelling. Occasionally, it may present with a discharging sinus. Constitutional symptoms may or may not be present. Clinically, the findings with restricted shoulder movements may mimic frozen shoulder and the diagnosis is delayed or missed. Erythrocyte sedimentation rate and C-reactive protein may be raised. Radiographs usually
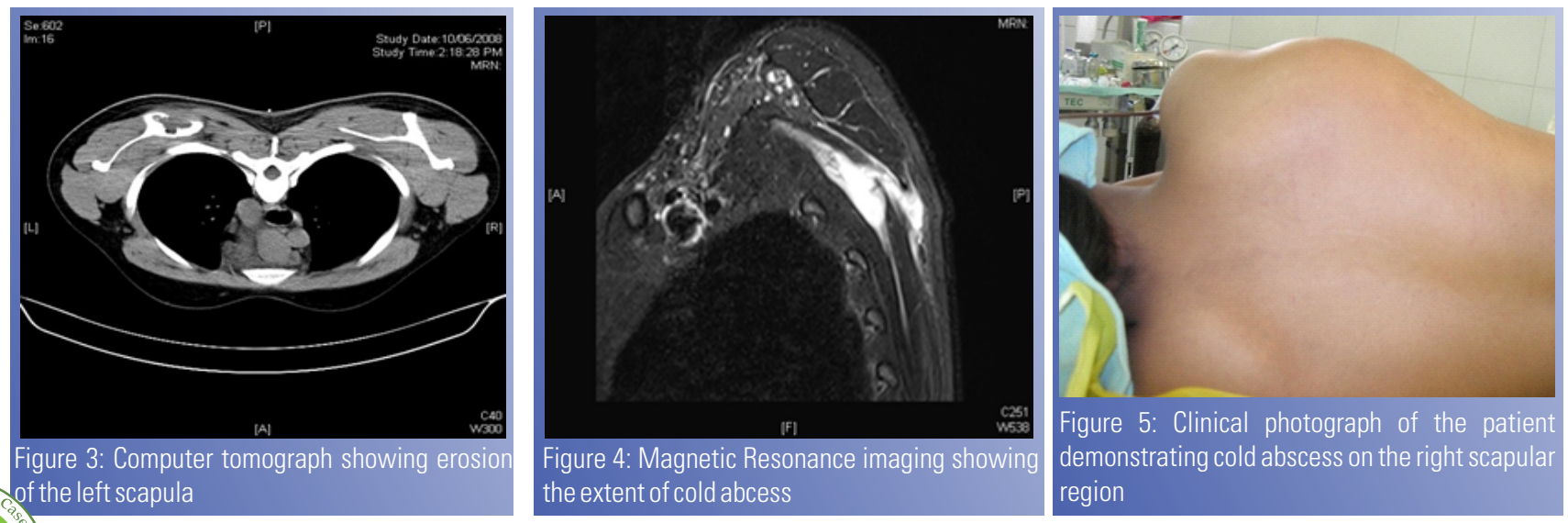

Journal of Orthopaedic Case Reports | Volume 3 | Issue 4 | Oct- Dec 2013 | Page 7-11 

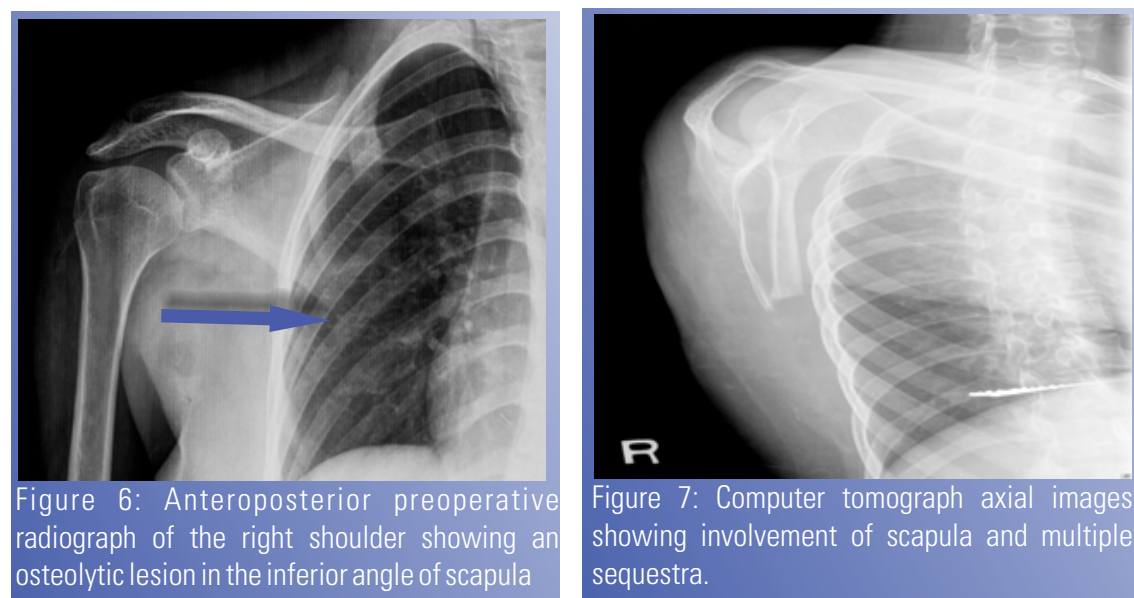

outcome and none had any complications. All of them were immunocompetent patients.

The main step in the diagnosis of skeletal tuberculosis is thorough clinical and radiological examination. From our analysis, tuberculosis of the scapula must be suspected if a patient presents with age less than 30 years, with pain and swelling in the scapular region less than six months duration and radiographs showing osteolytic lesion with surrounding marginal sclerosis. Though rare, tuberculosis should always be suspected in isolated scapular swelling in developing countries.

Antituberculous treatment usually gives excellent results . The abscess usually subsides on initiating the treatment. Surgery is advised only if there is giant sequestra or when there are no signs of improvement 3 to 4 weeks after initiating antitubercular therapy [1]. Our second case had multiple sequestra and a large cold abscess. Hence she underwent surgical debridement and sequestrectomy. Both patients showed dramatic improvement with nine months of therapy and showed complete resolution of the disease. Since all the patients reported so far with isolated involvement of scapula are immunocompetent, may be there was less chance of dissemination and none of them were multidrug resistant.

High index of suspicion is necessary to identify scapular involvement in all patients with shoulder pain. Thorough clinical and radiological examination is necessary to avoid misdiagnosis, as most shoulder problems are treated as periarthritis or frozen shoulder. Wasting of muscles is characteristic of tubercular involvement. Radiographs should be overlooked to identify any lesion in acromian, clavicle, scapula, coracoid process other than head of humerus. Bone scintigraphy and magnetic resonance imaging might be useful if in doubt. However, diagnosis can be established by needle aspiration or open biopsy.

\section{Conclusion}

To conclude, high index of suspicion is necessary for diagnosing this condition in endemic areas. Pain and swelling is the most common presentation of scapular tuberculosis. Radiography shows lytic lesion with marginal sclerosis. The prognosis is excellent with appropriate treatment. 


\section{Clinical Message}

Isolated scapular tuberculosis can be managed successfully with excellent prognosis if identified early

\section{References}

1. Singh A, Chatterjee P, Pai MC, Chacko RT: Tuberculous osteomyelitis of the scapula masquerading as metastasis. Radiology Case 2009, 3(1):27-31.

2. Mohan V, Danielsson L, Hosni G, Gupta RP: A case of tuberculosis of the scapula. Acta Orthop Scand 1991, 62:79-80.

3. Jain D, Jain VK, Singh Y, Kumar S, Mittal D: Cystic tuberculosis of the scapula in a young boy: a case report and review of the literature. J Med Case Reports 2009, 3:7412.

4. Tripathy SK, Sen RK, Sharma A, Tamuk T. Isolated cystic tuberculosis of scapula; case report and review of literature.J. Orthop Surg Res. 2010 Oct 8;5:72.

5. Guasti D, Devoti D, Affanni M: Tubercular osteitis of the spine of the scapula. Chir Organi Mov 1997, 82:413-418.

6. Vohra R, Kang HS, Dogra S, Saggar RR, Sharma R: Tuberculous osteomyelitis.JBone Joint Surg [Br] 1997, 79:562-566.

7. Kam WL, Leung YF, Chung OM, Wai YL: Tuberculous osteomyelitis of the scapula. Int Orthop 2000, 24:301-302.

8. Husen YA, Nadeem N, Aslam F, Shah MA: Tuberculosis of the scapula. JPak Med Assoc 2006, 56:336-338.

9. Srivastava P, Srivastava S: Tuberculosis of the scapula.Indian J Surg 2006, 68:27-9.

10. Solav S: Correlative Imaging in Skeletal Tuberculosis with Special emphasis on Radionuclide Bone Scintigraphy: A Pictorial Essay. World Journal of Nuclear Medicine 2007, 6(1):19-28.
Conflict of Interest: Nil Source of Support: None
How to Cite this Article:

Balaji G, Arockiaraj J, Roy AC, Ashok A. Isolated Tubercular Osteomyelitis of Scapula - A Report of Two Cases and Review of Literature. Journal of Orthopaedic Case Reports 2013 Oct-Dec;3(4): 7-11 